\title{
Generation of Emission Centers for Broadband NIR Luminescence in Bismuthate Glass by Femtosecond Laser Irradiation
}

\author{
Mingying Peng, ${ }^{\dagger \ddagger}$ Quanzhong Zhao, ${ }^{\S}$ Jianrong Qiu, ${ }^{\circledR}$ and Lothar Wondraczek ${ }^{\ddagger}$ \\ ${ }^{\ddagger}$ Department of Materials Science - WW3, University of Erlangen-Nuremberg, Erlangen 91058, Germany \\ ${ }^{\S}$ Institute of Optics, Information and Photonics, Max Planck Research Group, University of Erlangen-Nuremberg, \\ Erlangen 91058, Germany \\ 'State Key Laboratory of Silicon Materials, Zhejiang University, Hangzhou 310027, China
}

\begin{abstract}
Bismuth-doped glasses have recently received significant interest as potential material for ultrabroadband optical amplification in the telecommunication spectral bands, as well as as gain material for fiber lasers. However, the nature of the active centers that are responsible for the observed near-infrared (NIR) luminescence is still highly debated. In order to probe the mechanism that leads to NIR emission in bismuth-containing glasses, femtosecond (fs) laser irradiation was used. It is shown that local absorption properties in the visible spectral range can be altered in initially transparent bismuthate glasses after fs laser irradiation. Induced absorption centers exhibit the well-known broadband optical emission peaking at $\sim 1250 \mathrm{~nm}$ when excited with a $785 \mathrm{~nm}$ diode laser. Absorption and emission intensities increase with increasing average pulse energy. These observations are interpreted as a photo-induced reduction reaction of $\mathrm{Bi}^{3+}$ to $\mathrm{Bi}^{+}$ species, while the previously discussed formation of Bi-clusters by ion diffusion is excluded due to the very short interaction time that results from the use of fs laser. $\mathrm{Bi}^{+}$species are, therefore, proposed as the major origin of NIR emission from Bi-doped glasses.
\end{abstract}

\section{Introduction}

$\mathrm{R}$ APID growth in data capacity of telecommunication networks continues to stimulate exploration of luminescent materials (glasses) with improved emission bandwidth in the near-infrared (NIR) spectral range. In the future, such materials are expected to provide a new generation of broadband optical fiber amplifiers: the larger the bandwidth of the optical amplifier, the more transmission channels can be accessed, and the more data can be transferred simultaneously in a single optical fiber. ${ }^{1-5}$ In this context, Bi-doped glasses have experienced extensive research efforts. ${ }^{2-8}$ Under a variety of excitation conditions, such glasses exhibit NIR emission in the range of 1000 $1700 \mathrm{~nm}$ with bandwidths varying between $\sim 200$ and $500 \mathrm{~nm}$. Fluorescence occurs with lifetimes of up to several hundred microseconds. ${ }^{3-8}$ After the first report by Murata et al., ${ }^{2}$ NIR emission has now been observed in silicate, ${ }^{4,5,7-9}$ germanate, ${ }^{3,6,10}$ borate, ${ }^{11}$ phosphate, ${ }^{12,13}$ and chalcogenide ${ }^{14}$ glasses. All these glasses have been proposed as gain medium for ultrabroadband fiber amplifiers. ${ }^{2-14}$ In subsequent experiments, optical amplification, laser output, tunable laser operation, and all-fiber lasers were demonstrated based on Bi-doped glasses. ${ }^{15-18}$ More re-

S. Bhandarkar-contributing editor

\footnotetext{
Manuscript No. 25333. Received October 8, 2008; approved November 18, 2008.

Author to whom correspondence should be addressed. e-mail: mingying.peng@ ww.uni-erlangen.de
}

cently, Bi-doped glasses were further considered for use as spectral converters and planar luminescent concentrators for photovoltaic energy conversion using low-bandgap semiconductors. ${ }^{19}$ On the other hand, the origin of NIR emission still remains highly debated. It is now widely accepted that $\mathrm{Bi}^{3+}$ and $\mathrm{Bi}^{2+}$ ions do not contribute to NIR emission. ${ }^{3-5,7-15}$ Several proposals have been made tentatively, assigning the role of emission center to $\mathrm{Bi}^{5+7,20} \mathrm{Bi}^{+}, 11-15,21$ and even $\mathrm{Bi}$-ion clusters. ${ }^{10,22}$ Fujimoto and colleagues ${ }^{7,23}$ suggested $\mathrm{Bi}^{5+}$ ions as NIR emission centers and confirmed the presence of $\mathrm{Bi}^{5+}$ species in NIR-luminescent Bi-doped alumino-silicate glasses by X-ray absorption fine structure analysis. However, other authors, ${ }^{9,11}$ showed that increasing basicity of the host glass (what generally favors the higher valence states of $\mathrm{Bi}$ ) resulted in disappearing NIR emission. Furthermore, introducing reducing agents such as carbon into the batch, Arai et al. ${ }^{24}$ clearly demonstrated improved emission intensity. Truong et al. ${ }^{21}$ found similar results when heat-treating Bi-doped silicate glass in $\mathrm{H}_{2}$ atmosphere. These experimental results point to $\mathrm{Bi}^{+}$as the responsible emission center. Other opinions are based on the fact that at high temperatures, $\mathrm{Bi}_{2} \mathrm{O}_{3}$ can dissociate and convert into $\mathrm{BiO}$ or even into $\mathrm{Bi}$ metal. Then, Bi clusters may form by aggregation of $\mathrm{Bi}$ atoms. ${ }^{10,22}$

Femtosecond (fs) ultrashort pulse lasers are powerful tools for fabricating different functional optical devices, such as optical waveguides, couplers, photonic crystals, 3D optical storage devices, and rewritable optical memory, because they can in a controlled way induce various microstructural changes in transparent materials. ${ }^{25,26}$ For example, ${ }^{25}$ fs laser irradiation has been applied to $\mathrm{Sm}^{3+}$-doped alumino-borate glass to selectively and permanently reduce $\mathrm{Sm}^{3+}$ to $\mathrm{Sm}^{2+}$. If such a glass was used as an optical storage device, data could be read-out in form of luminescence with a very high signal-to-noise ratio. As another example, Qiu et al. ${ }^{26}$ developed a method to space-selectively precipitate gold nanoparticles in $\mathrm{Au}^{3+}$-doped silicate glass by combining fs laser irradiation and successive annealing, and they proposed to produce 3D Au nanocircuits in $\mathrm{Au}^{3+}$-doped glass by utilizing this scheme.

In the following, the interaction between fs laser pulses and $\mathrm{Bi}^{3+}$ containing glass will be addressed for two reasons: (a) bismuthate glasses could provide a competitive alternative as base material for 3D laser writing of colored structures and, (b) such experiments may, indirectly, help to deduce the mechanism that leads to NIR luminescence in similar glasses.

\section{Experimental Procedure}

Glass samples were synthesized by conventional melting and quenching. Analytic-grade reagents $\mathrm{H}_{3} \mathrm{BO}_{3}, \mathrm{SiO}_{2}, \mathrm{SrCO}_{3}$, $\mathrm{Sb}_{2} \mathrm{O}_{3}, \mathrm{PbO}, \mathrm{Al}(\mathrm{OH})_{3}$, and $\mathrm{Bi}_{2} \mathrm{O}_{3}$ were selected as raw materi- 
als. Approximately $60 \mathrm{~g}$ of a bismuthate glass with composition (in $\mathrm{wt} \%$ ) $52 \mathrm{Bi}_{2} \mathrm{O}_{3} \cdot 23 \mathrm{~B}_{2} \mathrm{O}_{3} \cdot 4 \mathrm{SiO}_{2} \cdot 17 \mathrm{PbO} \cdot 4 \mathrm{Sb}_{2} \mathrm{O}_{3}$ (BBSPS) were melted in an alumina crucible at $1200^{\circ} \mathrm{C}$ for $30 \mathrm{~min}$, cast onto a stainless-steel plate immediately after melting, and finally annealed at $300^{\circ} \mathrm{C}$ for $1 \mathrm{~h}$. Obtained samples were cut and polished into suitable shapes for optical analyses. Optical absorption spectra were recorded using a JASCO V-570 spectrophotometer (JASCO International Co. Ltd., Japan), and infrared fluorescence spectra were determined using the set-up as follows: for excitation, a $100 \mathrm{~mW} / 785 \mathrm{~nm}$ laser diode was used; emitted light was introduced into a monochromator equipped with three gratings $(1200 \mathrm{G} / \mathrm{mm}$ for $200-600 \mathrm{~nm} ; 600 \mathrm{G} / \mathrm{mm}$ for 500-1500 nm; $300 \mathrm{G} / \mathrm{mm}$ for $800-2500 \mathrm{~nm}$ ) and collected by an InGaAs detector. The current signal from the detector was transformed to a voltage signal by an I-V converter and transferred to a lock-in amplifier that was triggered by an optical chopper operating in the beamline at $80 \mathrm{~Hz}$. Fluorescence decay curves were obtained using a digital oscilloscope $(500 \mathrm{MHz}$ Tektronix TDS3054, Tektronix GmbH, Germany). All measurements were carried out at room temperature.

In order to study the interaction between fs laser pulses and the BBSPS glass, a commercial mode-locked multipass amplified Ti:sapphire laser system (Femtopower Compact PRO, Femtolaser Produktions $\mathrm{GmbH}$, Germany) with emission at $800 \mathrm{~nm}$, pulse duration of $33 \mathrm{fs}$, and a repetition rate of $1 \mathrm{kHz}$ was used. A variable neutral density filter was used to control pulse energy. The laser beam with an average pulse energy of $2.5 \mu \mathrm{J}$ (or $1 \mu \mathrm{J}$ ) was focused into the glass sample with a $\times 10$ lens $(\mathrm{NA}=0.3)$ with the help of a computer controlled XYZ translation stage. In order to measure absorption and emission spectra of the glass after laser irradiation, a plane of $2.5 \mathrm{~mm} \times 2.5 \mathrm{~mm}$ was written into the sample, consisting of damaged lines at an interval of 10 $\mu \mathrm{m}$, by scanning the laser beam at a rate of $1 \mathrm{~mm} / \mathrm{s}$. The distance of the plane from the surface of the glass sample was $1 \mathrm{~mm}$.

\section{Results}

Figure 1 shows the absorption spectra of BBSPS glass samples before and after fs pulse laser irradiation. In the as-received glass, very high transparency is observed in the visible range from 400 to $800 \mathrm{~nm}$. The glass appears weakly yellow due to the presence of $\mathrm{Bi}^{3+}$. Irradiation caused apparition of a strong absorption peak at around $500 \mathrm{~nm}$, and peak intensity increased as the average pulse energy was increased from 1.0 to $2.5 \mu \mathrm{J}$ (Figs.

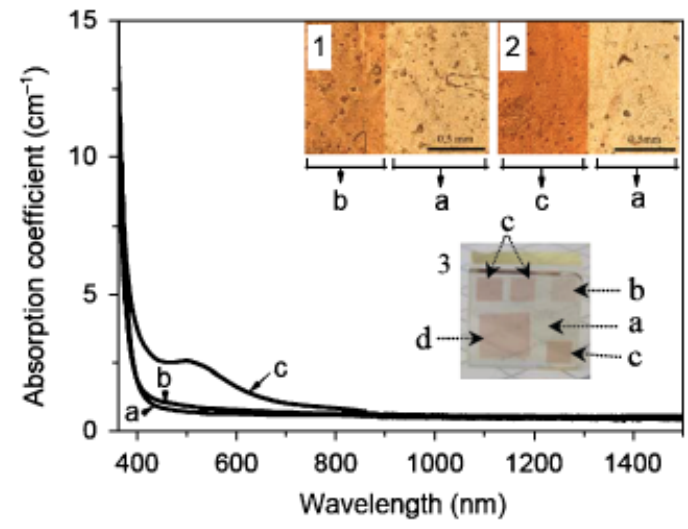

Fig. 1. Absorption spectra of BBSPS glasses before femtosecond (fs) laser illumination (a), after fs laser illumination with average pulse energy of $1 \mu \mathrm{J}(\mathrm{b})$, and $2.5 \mu \mathrm{J}(\mathrm{c})$, respectively. Insets 1 and 2 show samples after fs laser irradiations with average pulse energy of 1 and $2.5 \mu \mathrm{J}$, respectively. Scale bars are $0.5 \mathrm{~mm}$. Inset 3 is the photograph of the glass with fs laser-irradiated areas a-d. The $5 \mathrm{~mm} \times 5 \mathrm{~mm}$ area din inset 3 was written with fs laser with average pulse energy of $2.5 \mu \mathrm{J}$ in March 2005 . The experiment of irradiating with fs laser with average pulse energy of $2.5 \mu \mathrm{J}$ was repeated two more times to demonstrate reproducibility. BBSPS, $52 \mathrm{Bi}_{2} \mathrm{O}_{3} \cdot 23 \mathrm{~B}_{2} \mathrm{O}_{3} \cdot 4 \mathrm{SiO}_{2} \cdot 17 \mathrm{PbO} \cdot 4 \mathrm{Sb}_{2} \mathrm{O}_{3}$.

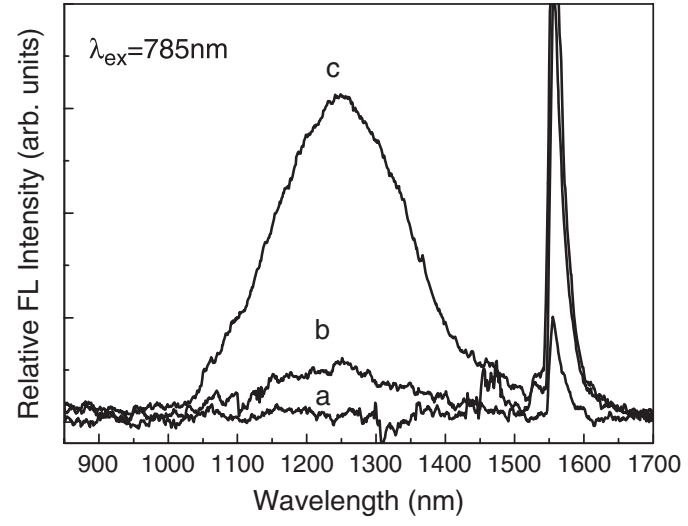

Fig. 2. Emission spectra of BBSPS glasses when pumped by $785 \mathrm{~nm}$ laser diode: (a) before femtosecond (fs) laser illumination, (b) after $1 \mu \mathrm{J}$ fs laser irradiation, and (c) after $2.5 \mu \mathrm{J}$ fs laser irradiation. BBSPS, $52 \mathrm{Bi}_{2} \mathrm{O}_{3} \cdot 23 \mathrm{~B}_{2} \mathrm{O}_{3} \cdot 4 \mathrm{SiO}_{2} \cdot 17 \mathrm{PbO} \cdot 4 \mathrm{Sb}_{2} \mathrm{O}_{3}$.

1(b) and (c)). Correspondingly, in areas where the glass was irradiated, it turned pink (inset of Fig. 1). The experiment of irradiating by $2.5 \mu \mathrm{J}$ fs pulse laser was repeated two more times to prove reproducibility (inset of Fig. 1).

NIR emission spectra of BBSPS glass samples are shown in Fig. 2. While in as-received glass, no NIR emission can be detected after excitation (785 nm laser diode, Fig. 2(a)), in the fs laser-irradiated area of the sample $(1.0 \mu \mathrm{J}$, area of inset of Fig. 1(b)), a weak emission peak protrudes in the range of $1000-1500 \mathrm{~nm}$ (Fig. 2(b)). When increasing the average energy to $2.5 \mu \mathrm{J}$, the peak position remains unchanged, but its intensity is enhanced by a factor of $\sim 6$ (Fig. 2(c), and area of inset of Fig. 1(c)).

Figure 3 is a representation of the decay curve of the emission at $1250 \mathrm{~nm}$. For comparison, data were fitted to a first order exponential decay equation, $y=1.00475 \mathrm{e}^{-x / 179}+0.02395$, which produced a fluorescence lifetime of $179 \mu$ s.

\section{Discussion}

Bismuthate (Bi-rich) glasses are long known for several applications, e.g. sealing glasses, glazes, etc. If melted in air, in such glasses bismuth is usually present in the form of $\mathrm{Bi}^{3+}$, and glasses appear yellowish. When illuminated with UV-light $(\sim 230-250 \mathrm{~nm})$, low concentrations of $\mathrm{Bi}^{3+}$ may result in emission of blue light $(\sim 450 \mathrm{~nm})$, with very short fluorescence decay times of $<10 \mu \mathrm{s} .{ }^{27}$ The observed emission properties of fs laserirradiated BBSPS glass are similar to those of other Bi-doped glasses, ${ }^{2-14,22,24}$ which leads to the conclusion that in the considered glass, fs laser-induced NIR emission originates from the same emission centers as it does in other Bi-doped glasses.

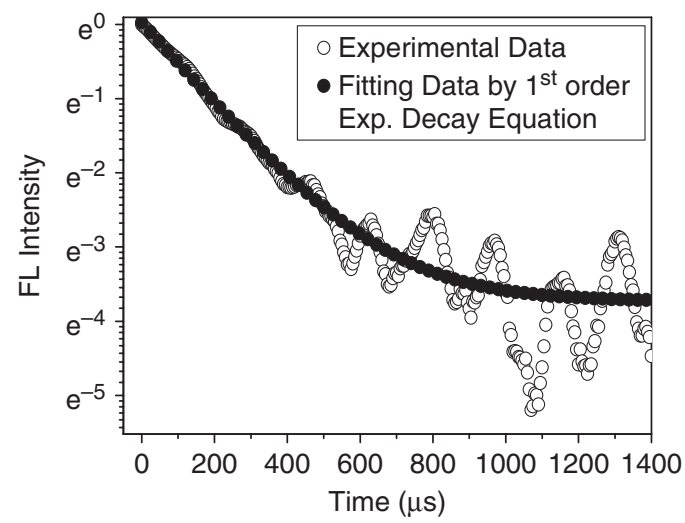

Fig. 3. Fluorescent decay curve of BBSPS glass irradiated by femtosecond $800 \mathrm{~nm}$ pulse laser with average pulse energy of $2.5 \mu \mathrm{J}$ when pumped by $785 \mathrm{~nm}$ laser diode. The monitored emission wavelength is $1250 \mathrm{~nm}$. BBSPS, $52 \mathrm{Bi}_{2} \mathrm{O}_{3} \cdot 23 \mathrm{~B}_{2} \mathrm{O}_{3} \cdot 4 \mathrm{SiO}_{2} \cdot 17 \mathrm{PbO} \cdot 4 \mathrm{Sb}_{2} \mathrm{O}_{3}$. 
Given the pulse width of $\sim 33$ fs and the average pulse energy of $1 \mu \mathrm{J}$ of the used laser, a power density of up to $3.8 \times 10^{13} \mathrm{~W} /$ $\mathrm{cm}^{2}$ results in the focal point of irradiation. In most glasses, this is sufficient to induce various nonlinear physicochemical reactions. For instance, the already mentioned fs laser-induced photoreduction of $\mathrm{Au}^{3+}$ to $\mathrm{Au}$ in $\mathrm{Au}^{3+}$-doped silicate glass ${ }^{26}$ and $\mathrm{Sm}^{3+} \rightarrow \mathrm{Sm}^{2+}$ in $\mathrm{Sm}^{3+}$-doped alumino-borate glass ${ }^{25}$ were attributed to multiphoton absorption processes. In the case of Au-containing silicate glasses, additional thermal treatment is necessary to obtain the desired 3D coloration effects by diffusion-driven formation of metallic clusters. Typically, the desired gold particles form while heat-treating $0.5-1 \mathrm{~h}$ at $550^{\circ} \mathrm{C}{ }^{26}$ Therefore, the present observation that NIR emission (as well as changes in the absorption spectrum) occur directly after fs laser irradiation (i.e., no additional thermal treatment is required) enables an important conclusion on the underlying emission mechanism.

The focal spot of the laser beam has a diameter of $\sim 10 \mu \mathrm{m}$. In view of the scanning speed of $1 \mathrm{~mm} / \mathrm{s}$ and the repetition rate of $1 \mathrm{kHz}$, the number of laser pulses applied to each focal point can be calculated as $\sim 10$. This means that the interaction time is about 330 fs between fs laser and BBSPS glass. Although quantitative data on diffusion is not available in sufficient detail, some conclusions can be drawn from comparison with $\mathrm{Ag}$ diffusion. Atomic radii of silver and bismuth are comparable. Effective ionic radii of six-fold coordinated $\mathrm{Bi}^{3+}$ (here, we refer to $\mathrm{Bi}^{3+}$ because data for $\mathrm{Bi}^{+}$are not available; note that the effective radius of $\mathrm{Bi}^{+}$would be even higher than that of $\mathrm{Bi}^{3+}$ ) and $\mathrm{Ag}^{+}$are 103 and $125 \mathrm{pm},{ }^{28}$ respectively. An order-of-magnitude of the diffusion coefficient of $\mathrm{Ag}, D_{0}=4.7 \times 10^{-2} \mathrm{~cm}^{2} / \mathrm{s}$ and the corresponding activation energy of diffusion, $E_{\mathrm{A}}=161$ $\mathrm{kJ} / \mathrm{mol}$, can be taken from Euken. ${ }^{29}$ This results in an estimated diffusion coefficient $6.2 \times 10^{-13} \mathrm{~cm}^{2} / \mathrm{s}\left(6.2 \times 10^{-28} \mathrm{~cm}^{2} / \mathrm{fs}\right)$ at $500^{\circ} \mathrm{C}$. This means that during the estimated interaction time of $\sim 330 \mathrm{fs}$, virtually no diffusion occurs at a length scale $>1 \mathrm{~nm}$. Instead, it is assumed that the observed effects are based on fast photo-induced reduction of $\mathrm{Bi}^{3+}$ to $\mathrm{Bi}^{+}$. At sufficiently high laser intensity, electrons simultaneously absorb multiple photons that allow them to pass from valence to conduction band. Once an electron is promoted to the conduction band, it will nucleate a process of avalanche ionization: by impact ionization, excess energy may be transferred to another electron, resulting in two electrons in the conduction band. Increasing the pulse energy leads to an increase of the number of these electrons and thereby improves the probability of permanent reduction, resulting in increasing concentration of $\mathrm{Bi}^{+}$and, consequently, increasing NIR emission intensity (Figs. 1 and 2).

It is further noteworthy that the fs laser-induced coloration does not, at ambient temperature, fade with time; some irradiated samples have been stored for more than 3 years (since March 2005 ) without any visible alteration of laser-written features (such as area in inset 3 of Fig. 1(d)). Compared with expensive $\mathrm{Au}^{3+}$. or $\mathrm{Ag}^{+}$-doped glasses, where fs pulse lasers in combination with subsequent heat treatment have been used to space-selectively precipitate gold or silver nanoparticles to produce colored images in transparent glass blocks, $\mathrm{Bi}^{3+}$-doped glasses where the bismuth ions can be reduced to $\mathrm{Bi}^{+}$may offer a convenient alternative. Similarly colorful structures can be written into such glasses, but without any additional thermal treatment.

\section{Conclusions}

For the first time, it was demonstrated that fs laser irradiation can be used to write colored features into bismuthate glasses. Upon optical excitation, irradiated areas exhibit NIR emission, peaking at $\sim 1250 \mathrm{~nm}$. Induction of coloration and NIR emission do not require thermal annealing subsequent to laser irradiation. Emission properties are similar to these of other Bi-doped glasses. It is concluded that, thus, emission occurs from the same emission centers. Because of the short interaction time between fs laser and glass, diffusion-driven formation of
Bi-containing clusters is excluded. Rather, it is concluded that $\mathrm{Bi}^{+}$species are responsible for NIR emission in this class of materials. Laser irradiation results in photo-induced reduction of $\mathrm{Bi}^{3+}$ to $\mathrm{Bi}^{+}$by multiphoton absorption. It is suggested that for the fabrication of colored, 3D structures in transparent glasses via fs laser writing, $\mathrm{Bi}^{3+}$-containing glasses offer a convenient alternative for $\mathrm{Au}^{3+}$ or $\mathrm{Ag}^{+}$-doped glasses.

\section{References}

${ }^{1}$ T. Suzuki and Y. Ohishi, "Broadband $1400 \mathrm{~nm}$ Emission from $\mathrm{Ni}^{2+}$ in ZincAlumino-Silicate Glass,” Appl. Phys. Lett., 84 [19] 3804-6 (2004).

${ }^{2}$ K. Murata, Y. Fujimoto, T. Kanabe, H. Fujita, and M. Nakatsuka, "Bi-Doped $\mathrm{SiO}_{2}$ as a New Laser Material for an Intense Laser," Fusion Eng. Des., 44, 437-9 (1999).

${ }^{3}$ M. Peng, X. Meng, J. Qiu, and Q. Zhao, "GeO $: B i$, M $(\mathrm{M}=\mathrm{Ga}$, B) Glasses with Super-Wide Infrared Luminescence,” Chem. Phys. Lett., 403, 410-4 (2005).

${ }^{4}$ M. Peng, X. Meng, D. Chen, and J. Qiu, "Comment on "Enhanced RoomTemperature Emission in $\mathrm{Cr}^{4+}$ Ions Containing Alumino-Silicate Glasses" [Appl. Phys. Lett. 82, 4035 (2003)]," Appl. Phys. Lett., 87 [6] 066103-1-2 (2005).

${ }^{5}$ M. Peng, J. Qiu, D. Chen, X. Meng, and C. Zhu, "Broadband Infrared Luminescence from $\mathrm{Li}_{2} \mathrm{O}-\mathrm{Al}_{2} \mathrm{O}_{3}-\mathrm{ZnO}-\mathrm{SiO}_{2}$ Glasses Doped with $\mathrm{Bi}_{2} \mathrm{O}_{3}$," Opt. Express, 13, 6892-8 (2005).

${ }^{6}$ M. Peng, J. Qiu, D. Chen, X. Meng, L. Yang, X. Jiang, and C. Zhu, "Bismuthand Aluminum-Codoped Germanium Oxide Glasses for Super-Broadband Optical Amplification," Opt. Lett., 29, 1998-2000 (2004).

${ }^{7}$ Y. Fujimoto and M. Nakatsuka, "Infrared Luminescence from BismuthDoped Silica Glass,” Jpn. J. Appl. Phy., 40, L279-81 (2001).

${ }^{8}$ T. Suzuki and Y. Ohishi, "Ultrabroadband Near-Infrared Emission from BiDoped $\mathrm{Li}_{2} \mathrm{O}-\mathrm{Al}_{2} \mathrm{O}_{3}-\mathrm{SiO}_{2}$ Glass," Appl. Phys. Lett., 88, 191912-1-3 (2006).

${ }^{9}$ M. Peng, C. Wang, N. Da, D. Chen, X. Jiang, C. Zhu, and J. Qiu, "BismuthActivated Luminescent Materials for Broadband Optical Amplifier in WDM System," J. Non-Cryst. Solids, 354, 1221-5 (2008).

${ }^{10}$ M. Peng, J. Qiu, D. Chen, X. Meng, and C. Zhu, "Superbroadband $1310 \mathrm{~nm}$ Emission from Bismuth and Tantalum Codoped Germanium Oxide Glasses," Opt. Lett., 30, 2433-5 (2005)

${ }^{11}$ X. Meng, J. Qiu, M. Peng, D. Chen, Q. Zhao, X. Jiang, and C. Zhu, "Infrared Broadband Emission of Bismuth-Doped Barium-Aluminum-Borate Glasses," Opt. Express, 13 [5] 1635-42 (2005).

${ }^{12}$ X. Meng, J. Qiu, M. Peng, D. Chen, Q. Zhao, X. Jiang, and C. Zhu, "Near Infrared Broadband Emission of Bismuth-Doped Aluminophosphate Glass," Opt. Express, 13 [5] 1628-34 (2005).

${ }^{13}$ B. Denker, B. Galagan, V. Osiko, S. Sverchkov, and E. Dianov, "Luminescent Properties of Bi-Doped Boro-Alumino-Phosphate Glasses," Appl. Phys. B, 87, 135-7 (2007).

${ }^{14}$ J. Ren, D. Chen, G. Yang, Y. Xu, H. Zeng, and G. Chen, "Near Infrared Broadband Emission from Bismuth-Dysprosium Codoped Chalcohalide Glasses," Chin. Phys. Lett., 24, 1958-60 (2007).

${ }^{15}$ E. Dianov, V. Dvoyrin, V. Mashinsky, A. Umnikov, M. Yashkov, and A. Gur'yanov, "CW Bismuth Fibre Laser," Quant. Electron., 35, $1083-4$ (2005).

${ }^{16}$ V. Dvoyrin, V. Mashinsky, I. Bulatov, A. Shubin, M. Melkumov, E. Kustov, E. Dianov, A. Umnikov, V. Khopin, M. Yashkov, and A. Guryanov, "BismuthDoped-Glass Optical Fibers-A New Active Medium for Lasers and Amplifiers," Opt. Lett., 31, 2966-8 (2006)

${ }^{17}$ I. Razdobreev, L. Bigot, V. Pureur, A. Favre, G. Bouwmans, and M. Douay, "Efficient All-Fiber Bismuth-Doped Laser," Appl. Phys. Lett., 90, 031103-1-3 (2007).

${ }^{18}$ E. Dianov, A. Shubin, M. Melkumov, O. Medvedkov, and I. Bufetov, "HighPower CW Bismuth-Fiber Lasers," J. Opt. Soc. Am. B, 24, 1749-55 (2007).

${ }^{19}$ M. Peng and L. Wondraczek, "Bismuth-Doped Oxide Glasses as Potential Solar Spectral Converters and Concentrators," J. Mater. Chem., (2009), DOI: $10.1039 / \mathrm{b} 812316 \mathrm{k}$.

${ }^{20}$ T. Ohkura, Y. Fujimoto, M. Nakatsuka, and S. Young-Seok, "Local Structures of Bismuth Ion in Bismuth-Doped Silica Glasses Analyzed Using Bi LIII X-Ray Absorption Fine Structure," J. Am. Ceram. Soc., 90, 3596-600 (2007).

${ }^{21}$ V. Truong, L. Bigot, A. Lerouge, M. Douay, and I. Razdobreev, "Study of Thermal Stability and Luminescence Quenching Properties of Bismuth-Doped Silicate Glasses for Fiber Laser Applications," Appl. Phys. Lett., 92, 041908-1-3 (2008)

${ }^{22}$ S. Khonthon, S. Morimoto, Y. Arai, and Y. Ohishi, "Luminescence Characteristics of Te- and Bi-Doped Glasses and Glass-Ceramics," J. Ceram. Soc. Jpn. 115, 259-63 (2007).

${ }^{23}$ T. Ohkura, Y. Fujimoto, M. Nakatsuka, and S. Young-Seok, "Local Structures of Bismuth Ion in Bismuth-Doped Silica Glasses Analyzed Using Bi LIII X-Ray Absorption Fine Structure," J. Am. Ceram. Soc., 90, 3596-600 (2007).

${ }^{24}$ Y. Arai, T. Suzuki, and Y. Ohishi, "Ultrabroadband Near-Infrared Emission from a Colorless Bismuth-Doped Glass," Appl. Phys. Lett., 90, 261110-1-3 (2007).

${ }^{25}$ J. Qiu, K. Miura, T. Suzuki, T. Mitsuyu, and K. Hirao, "Permanent Photoreduction of $\mathrm{Sm}^{3+}$ to $\mathrm{Sm}^{2+}$ Inside a Sodium Aluminoborate Glass by an Infrared Femtosecond Pulsed Laser," Appl. Phys. Lett., 74, 10-2 (1999).

${ }^{26}$ J. Qiu, X. Jiang, C. Zhu, M. Shirai, J. Si, N. Jiang, and K. Hirao, "Manipulation of Gold Nanoparticles Inside Transparent Materials," Angew. Chem. Int. Ed., 43, 2230-4 (2004)

${ }^{27}$ S. Parke and R. S. Webb, "The Optical Properties of Thallium, Lead and Bismuth in Oxide Glasses," J. Phys. Chem. Solids, 34, 85-95 (1973).

${ }^{28}$ R. Shannon, "Revised Effective Ionic Radii and Systematic Studies of Interatomie Distances in Halides and Chaleogenides," Acta Cryst., A32, 751-67 (1976).

${ }^{29}$ A. Euken, Landolt-Börnstein Zahlenwerte und Funktionen aus Physik, Chemie Astronomie, Geophysik und Technik. 6, Vol. I/4. Springer, Berlin, 1955. 